ARTICLE

https://doi.org/10.1038/s41467-019-11339-x

\title{
The MTR4 helicase recruits nuclear adaptors of the human RNA exosome using distinct arch-interacting motifs
}

\author{
Mahesh Lingaraju1, Dennis Johnsen², ${ }^{2}$, Andreas Schlundt 3,4,5,7, Lukas M. Langer (1) ', Jérôme Basquin', \\ Michael Sattler (10 ${ }^{3,4}$, Torben Heick Jensen ${ }^{2}$, Sebastian Falk (i) ${ }^{1,6}$ \& Elena Conti ${ }^{1}$
}

The nuclear exosome and its essential co-factor, the RNA helicase MTR4, play crucial roles in several RNA degradation pathways. Besides unwinding RNA substrates for exosomemediated degradation, MTR4 associates with RNA-binding proteins that function as adaptors in different RNA processing and decay pathways. Here, we identify and characterize the interactions of human MTR4 with a ribosome processing adaptor, NVL, and with ZCCHC8, an adaptor involved in the decay of small nuclear RNAs. We show that the unstructured regions of $\mathrm{NVL}$ and $\mathrm{ZCCHC8}$ contain short linear motifs that bind the MTR4 arch domain in a mutually exclusive manner. These short sequences diverged from the arch-interacting motif (AIM) of yeast rRNA processing factors. Our results suggest that nuclear exosome adaptors have evolved canonical and non-canonical AIM sequences to target human MTR4 and demonstrate the versatility and specificity with which the MTR4 arch domain can recruit a repertoire of different RNA-binding proteins.

\footnotetext{
${ }^{1}$ Department of Structural Cell Biology, Max-Planck-Institute of Biochemistry, Am Klopferspitz 18, D-82152 Martinsried, Germany. ${ }^{2}$ Department of Molecular Biology and Genetics, Aarhus University, C.F. Møllers Alle 3, 8000 Aarhus C, Denmark. ${ }^{3}$ Center for Integrated Protein Science Munich (CIPSM) at Department of Chemistry, Technical University of Munich (TUM), 85747 Garching, Germany. ${ }^{4}$ Institute of Structural Biology, Helmholtz-Zentrum München, 85764 Neuherberg, Germany. ${ }^{5}$ Present address: Institute for Molecular Biosciences and Center for Biomolecular Magnetic Resonance (BMRZ) at Johann Wolfgang Goethe-University, Frankfurt am Main 60438, Germany. ${ }^{6}$ Present address: Max F. Perutz Laboratories, Department of Structural and Computational Biology, University of Vienna, Campus Vienna Biocenter 5, 1030 Vienna, Austria. ${ }^{7}$ These authors contributed equally: Dennis Johnsen, Andreas Schlundt. Correspondence and requests for materials should be addressed to S.F. (email: sebastian.falk@univie.ac.at) or to E.C. (email: conti@biochem.mpg.de)
} 
E ukaryotic cells generate a multitude of RNA species that require timely maturation and decay to maintain a healthy transcriptome. A central machinery in nuclear RNA processing, quality control and decay pathways is a conserved $3^{\prime}-5^{\prime}$ exoribonuclease complex known as the RNA exosome (reviewed $\left.i^{1,2}\right)$. Most mechanistic studies to date have analyzed the RNA exosome from $S$. cerevisiae, the species in which it was originally identified 20 years ago $^{3}$. The yeast exosome consists of a 10subunit core complex $\left(\mathrm{Exo}_{10}\right)^{4}$, the activity of which depends on a single processive exoribonuclease (Rrp44, also known as Dis3 $)^{5,6}$. The $\mathrm{Exo}_{10}$ core is present in both nuclear and cytoplasmic compartments, but its cofactors and regulators have distinct subcellular localizations (reviewed in ${ }^{1,2}$ ). In the nucleus, $\mathrm{Exo}_{10}$ is bound to the distributive ribonuclease Rrp6 and its associated protein Rrp47 as well as to the Mpp6 protein $^{7-9}$. Together, Rrp6Rrp47 and Mpp6 recruit the RNA helicase Mtr4 to the exosome $e^{10-12}$. Orthologues of all these 14 proteins exist in human cells, and engage in similar interactions to form the corresponding human nuclear exosome complex ${ }^{12,13}$.

In both yeast and humans, the nuclear helicase Mtr4/MTR4 is central to exosome function ${ }^{2,14,15}$. First, it functions as an enzyme to remodel ribonucleoprotein (RNP) substrates with its $3^{\prime}-5^{\prime}$ unwinding activity, and to present the unwound RNA substrate to the exosome core ${ }^{12,16}$. Furthermore, it functions as a binding platform for RNA-binding adaptors, providing the primary interactions to transcripts subjected to exosomal degradation in both RNA processing and decay pathways ${ }^{17,18}$. For example, $S$. cerevisiae Mtr4 binds Nop53, a ribosome biogenesis factor that recruits the exosome for a late step in rRNA processing, namely the trimming of ITS2 (Internal Transcribed Spacer 2). Two other factors, Trf4 and Air2, bind Mtr4 to form the so-called TRAMP complex ${ }^{19-21}$, which allows the exosome to target aberrant tRNAs ${ }^{22}$, rRNAs and small nuclear and nucleolar RNAs (sn/ snoRNAs) for decay ${ }^{23}$. Higher eukaryotes not only have orthologues of Nop53 and TRAMP, but also have an increased number of nuclear exosome adaptors. In human cells, MTR4 has been reported to interact with the early ribosome biogenesis factors WDR74 and NVL, which take part in the processing of ITS1 (Internal Transcribed Spacer 1) 24,25 . Human MTR4 also binds to two large Zinc-finger proteins, ZCCHC8 and ZFC3H1. ZCCHC8 interacts with MTR4 and the RNA-binding protein RBM7 to form the trimeric Nuclear EXosome Targeting (NEXT) complex ${ }^{17,26}$, which targets enhancer RNAs (eRNAs), promoter upstream transcripts (PROMPTs) and intronic RNAs for exosome-mediated decay ${ }^{27}$. ZFC3H1 instead directs MTR4 and the nuclear exosome to polyadenylated nuclear RNAs by connecting to the nuclear poly $(\mathrm{A})$ binding protein $\mathrm{PABN} 1^{28-30}$. Furthermore, MTR4 binds NRDE-2, a negative regulator that prevents the nuclear exosome from targeting RNAs that should be exported to the cytoplasm ${ }^{31}$.

How does the nuclear exosome helicase mediate binding to such a diverse and functionally distinct set of proteins? Structural studies have shown that yeast Mtr4 contains an unstructured Nterminal region, a DExH helicase core and an 'arch' domain with a globular Kyprides, Ouzounis, Woese (KOW) domain ${ }^{14,32}$. All Mtr4 domains are involved in protein-protein interactions: the Nterminal region binds Rrp6-Rrp $47^{11}$, the helicase core binds Mpp6 as well as Trf4-Air $2^{33,34}$ and the KOW domain binds a short sequence known as 'arch-interacting motif that is present in Nop53, Utp18, and Air2 ${ }^{35,36}$. In the case of human MTR4, both the arch domain and the DExH helicase core bind NRDE$2^{31}$. Structural data have also elucidated how the helicase core of human MTR4 binds a region of $\mathrm{ZCCHC} 8^{37}$. This protein is however expected to harbor another MTR4-binding region ${ }^{37}$, which remains to be identified. Also unclear is how other human MTR4-binding proteins are recognized. With the exception of the expected AIM sequence in the human NOP53 orthologue (also known as GLTSCR2) and in NRDE- $2^{31}$, there is no consensus motif that can be identified with confidence at the sequence level in other MTR4-binding factors. Here, we used a combination of biochemical studies, X-ray crystallography and nuclear magnetic resonance (NMR) experiments to obtain mechanistic insights into how human MTR4 interacts with two metazoan RNA exosome adaptors: the RNA processing factor NVL and the RNA decay factor ZCCHC8.

\section{Results}

The unstructured region of human NVL interacts with MTR4. The nuclear VCP-like (NVL) protein, also known as NVL2, is a ribosome biogenesis factor of the AAA-ATPase family that has been reported to interact with human MTR4 ${ }^{38}$. NVL is a multidomain protein characterized by an $\mathrm{N}$-terminal nucleolin-binding domain (residues 10-74) 39 $^{3}$ and a linker region (residues 76-266) followed by two globular domains, characteristic of AAA-ATPases that are responsible for catalytic activity (Fig. 1a, Supplementary Fig. 1a). Bioinformatic analyses suggested that the linker region is mostly unstructured (residues 76-239) (Supplementary Fig. 1b). In other AAA-ATPases, unstructured regions upstream of the catalytic domains often mediate protein-protein interactions ${ }^{40-42}$. To test if the portion upstream of the NVL catalytic domains mediates the interaction with MTR4, we expressed and purified the N-terminal region of human NVL tagged with thioredoxin (Trx) as the prey protein (Trx-NVL $\left.{ }^{1-266}\right)$ and the structured portion of MTR4 tagged with glutathione-Stransferase (GST) as the bait protein (GST-MTR4- $\Delta N)$. In these assays, Trx-NVL ${ }^{1-266}$ specifically co-precipitated with GSTMTR4- $\Delta \mathrm{N}$ (Fig. 1b, lane 11, compare with GST control in lane 9). Interestingly, pull-down assays with the recombinant yeast orthologues showed that the N-terminal unstructured region of Rix7 does not interact with yeast Mtr4 (Supplementary Fig. 1c), indicating that the interaction between human NVL and MTR4 is not conserved in S. cerevisiae.

We narrowed down the MTR4-interacting region of human NVL based on bioinformatic analysis. Sequence alignments showed that the N-terminal unstructured region of NVL contains an insertion that is present in the human protein and other chordates but not in the yeast orthologue (Supplementary Fig. 1a). Upon testing whether this insertion is responsible for the interaction with human MTR4, we found that a construct encompassing the conserved portion of the human NVL insertion spanning residues 167-216 (Supplementary Fig. 1a) was indeed able to co-precipitate with GST-MTR4- $\Delta \mathrm{N}$ in pull-down assays (Fig. 1b, lane 14, Trx-NVL ${ }^{167-216}$ ). Thus, the MTR4-binding determinants of NVL reside in an unstructured region that is present in the human orthologue and more generally in chordate NVL proteins, but not in lower eukaryotes.

NVL targets the MTR4 KOW domain. To identify where the MTR4-binding determinants for NVL reside, we performed GST pull down assays with MTR4 constructs harboring the DExH core of the helicase (GST-MTR4 $\Delta \mathrm{N} \Delta \mathrm{arch}$ ) and the KOW domain (GST-MTR4 KOW). Neither Trx-NVL ${ }^{1-266}$ nor Trx-NVL ${ }^{167-216}$ co-precipitated with GST-MTR4- $\Delta \mathrm{N} \Delta$ arch (Fig. 1b, lanes 12 and 15). In contrast, the KOW domain characteristic of the MTR4 arch (GST-MTR4-KOW) was able to co-precipitate both TrxNVL $^{1-266}$ and Trx-NVL ${ }^{167-216}$ (Fig. 1b, lanes 13 and 16). We quantified the strength of the interaction by biophysical approaches. Using microscale thermophoresis (MST), we measured a dissociation constant of $\sim 1 \mu \mathrm{M}$ between a YFP-tagged version of NVL ${ }^{167-216}$ and MTR4-KOW (Fig. 1c). A similar dissociation constant was obtained when testing the interaction of NVL ${ }^{167-216}$ 
a
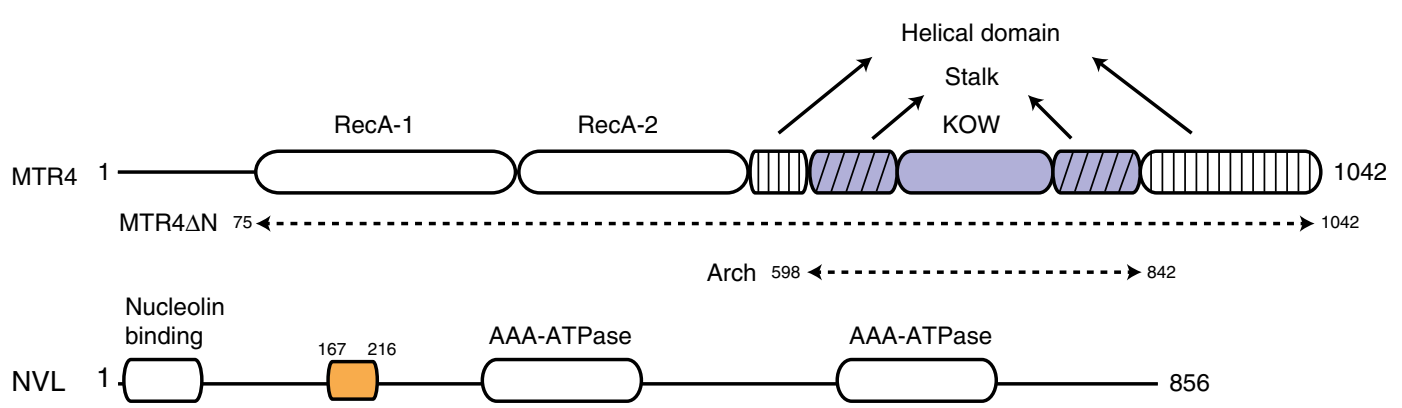

b
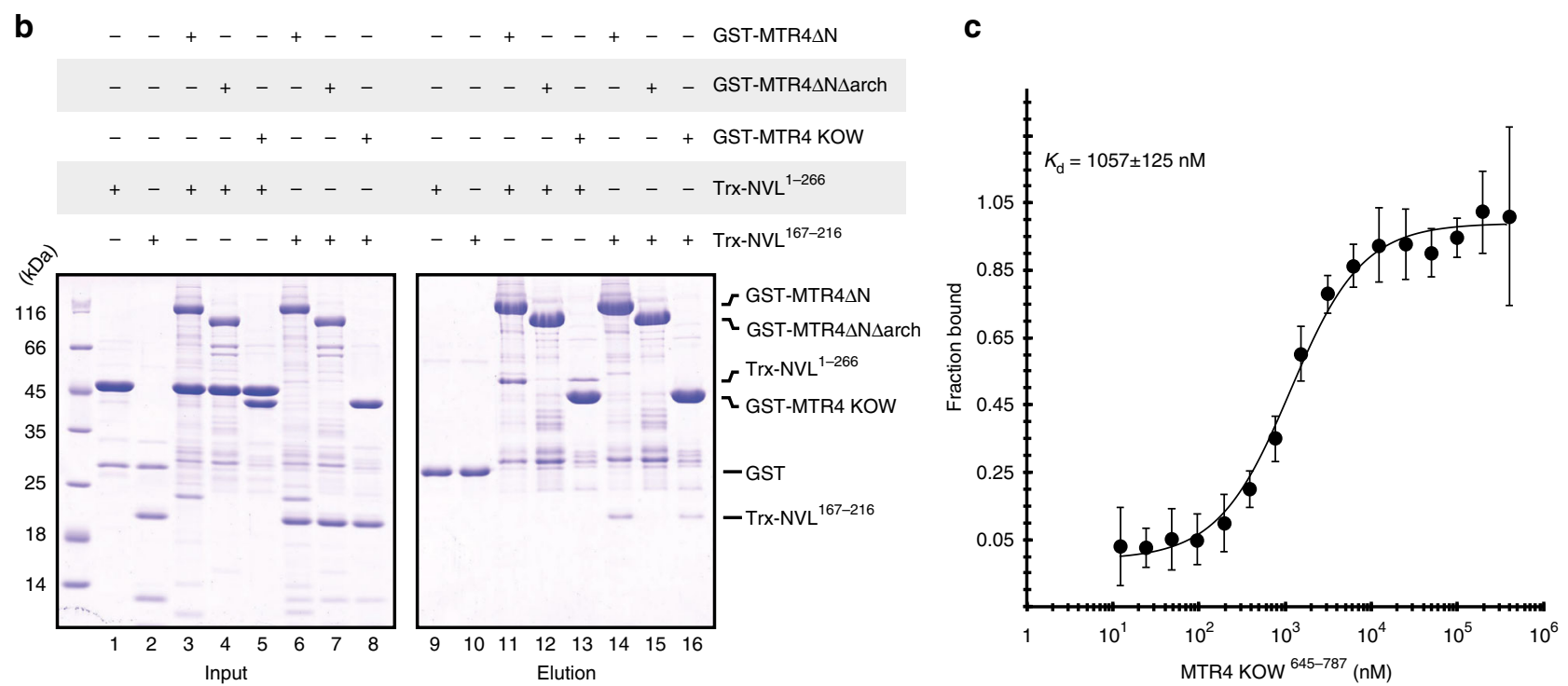

Fig. 1 The N-terminal unstructured region of NVL interacts with the MTR4 KOW. a Schematic representation of the domain organization of NVL and MTR4. Domain boundaries are obtained from previous studies ${ }^{31,37}$ and the crystal structure in the current report. The arrows indicate nomenclature for MTR4 constructs used in the text. b Protein co-precipitation assays. GST-tagged MTR4 $\Delta N$, MTR4 $\Delta N \Delta$ arch, and MTR4 KOW were incubated with either Trx-NVL ${ }^{1-266}$ or Trx-NVL ${ }^{167-216}$ before co-precipitation with glutathione sepharose beads. A total of $3 \%$ of the input (lanes 1-8) and $30 \%$ of the eluates (lanes 9-16) were analyzed on 15\% SDS-PAGE gels stained with coomassie brilliant blue. c Microscale thermophoresis experiment with MTR4 KOW and Trx-NVL 167-216_(GS) ${ }_{3}$-eYFP. In all, 50 nM of Trx-NVL 167-216-(GS) ${ }_{3}$-eYFP was incubated with increasing concentrations of MTR4 KOW and thermophoresis was measured by tracking the fluorescence of the NVL-YFP fusion protein. The binding isotherm was calculated using MO software (Nanotemper technologies) and the dissociation constant $\left(K_{d}\right)$ is given in the inset. The error bars represent the standard deviations of each data point calculated from three independent thermophoresis measurements

and MTR4- $\Delta \mathrm{N}$ using isothermal titration calorimetry (ITC) (Supplementary Fig. 1d). The fact that the KOW domain alone binds NVL ${ }^{167-216}$ as strongly as MTR4- $\Delta N$ indicated that the NVL ${ }^{167-216}$ binding region resides within the KOW domain.

We proceeded to characterize the NVL ${ }^{167-216}$-binding site of MTR4 with structural approaches. Using NMR spectroscopy, we confirmed that the MTR4-KOW domain has a sequential arrangement of secondary structure elements consistent with the fold observed in a recent crystal structure of an MTR4NRDE-2 complex ${ }^{31}$, namely a five-stranded $\beta$-sheet flanked by a long C-terminal helix and containing smaller helical segments within loops (Supplementary Fig. 2). We then carried out titration experiments using a ${ }^{15} \mathrm{~N}$-labelled MTR4-KOW protein and adding increasing amounts of unlabeled NVL ${ }^{167-216}$. In line with a dissociation constant of $\sim 1 \mu \mathrm{M}$, we observed an intermediate exchange regime for most of the peaks during the titration in HSQC (heteronuclear single quantum coherence) spectra. Chemical shift perturbations (CSP) measured upon NVL2 ${ }^{167-216}$ addition revealed significant effects (Fig. 2a-c) with clusters of strongly shifting peaks around residues 658, 695, 743, and 764. From the NMR analysis of the NVL ${ }^{167-216}$ - MTR4KOW interaction, the CSPs were very similar to those we had previously reported for the yeast Nop53-Mtr4 KOW interaction ${ }^{36}$ (Supplementary Fig. 3a, 3b). Consistently, a reverse-charge substitution of Arg743 (R743E mutant) impaired the binding of both NVL ${ }^{167-216}$ (pull-down assays in Fig. 2d, lane 8 ) and of the human Nop53 orthologue (Supplementary Fig. 3c).

Identification of W-AIM: a tryptophan arch-interacting motif of NVL. Given that NVL ${ }^{167-216}$ binds the KOW domain of MTR4 similarly to Nop53, we expected the presence of a similar AIM motif. A stretch of amino acids within this segment (NVL residues 185-190) appeared to show significant sequence similarity to the Nop53 AIM sequence (Fig. 3a). Surprisingly in this context, the F186A and D189R mutations in the Nop53-like stretch did not affect NVL ${ }^{167-216}$ binding to MTR4 in a pulldown assay with recombinant proteins (Fig. 3b, compare lanes 5 and 6), indicating that this segment is not a bona-fide AIM. To identify the arch-interacting motif in NVL ${ }^{167-216}$, we analyzed the sequences from different species in terms of evolutionary conservation. We noticed that vertebrates feature a conserved hydrophobic segment GWFIDKTP (residues 172-179, Fig. 3a; Supplementary Fig. 1a). Mutations in this segment (W173A, 
a

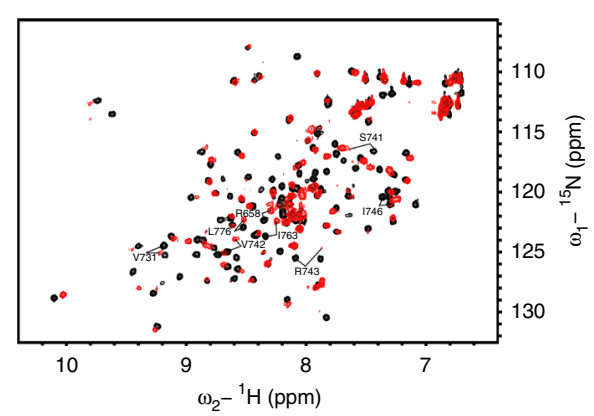

C

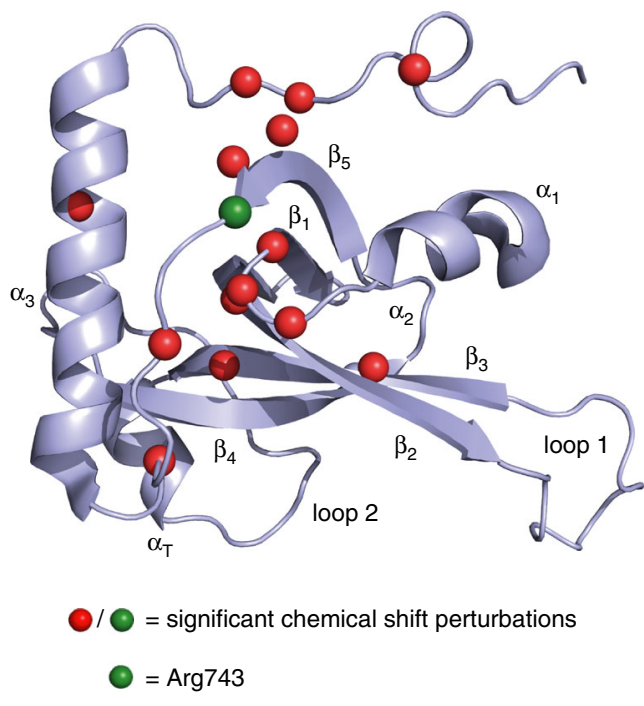

b
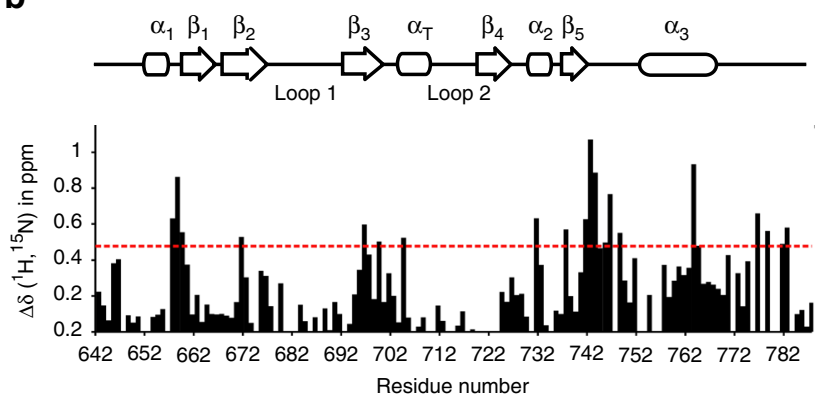

d

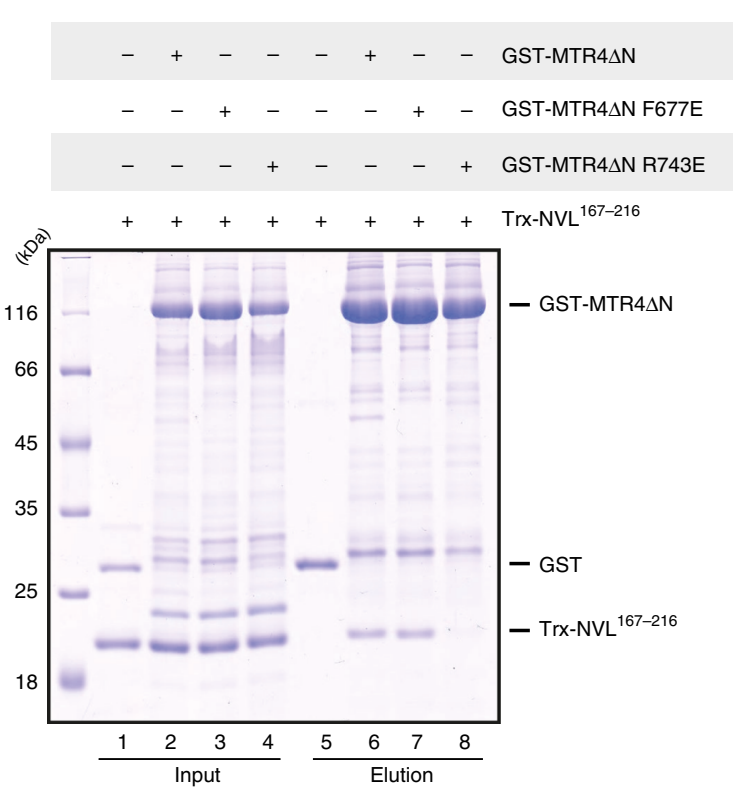

Fig. 2 Analysis of MTR4 KOW-NVL complex by NMR and site directed mutagenesis. a Overlay of ${ }^{1} \mathrm{H}-{ }^{15} \mathrm{~N}-\mathrm{HSQCs}$ of either MTR4 KOW alone (black) or in complex with a six-fold excess of NVL2 (red). Selected residues experiencing large chemical shift perturbations (CSPs) are labeled. b Plot of CSPs per residue of the MTR4 KOW sequence. The red line marks the threshold of significant CSPs, which are mapped on the model of MTR4 KOW (PDB 6IEH) in panel c. Gaps indicate either prolines or residues that could not be assigned. c MTR4 KOW domain (PDB 6IEH) with labeled secondary structural elements as assigned by NMR and residues showing significant CSPs (displayed as red/green spheres). $\mathbf{d}$ Protein co-precipitations by pull down assays. GST-tagged MTR4 $\triangle N$ (WT or mutants) were incubated with Trx-NVL ${ }^{167-216}$ before co-precipitation with glutathione sepharose beads. A total of $3 \%$ of the input (lanes 1-4) and 30\% of the eluates (lanes 5-8) were analyzed on 15\% SDS-PAGE gels and visualized by staining with coomassie brilliant blue

I175E) either abolished or impaired NVL ${ }^{167-216}$ binding to MTR $4 \Delta \mathrm{N}$ in pull-down assays in vitro (Fig. $3 \mathrm{~b}$, lanes 7 and 8 ), suggesting that this stretch functions as an arch-interacting motif, which we coin 'tryptophan-centered arch - interacting motif (WAIM).

We co-crystallized the NVL W-AIM peptide in complex with a construct of human MTR4 encompassing both the DExH core and the arch domain (residues 70-1042) (Table 1). The overall structure of the helicase is similar to that of the human NRDE-2MTR4 complex ${ }^{31}$, but with a notably different conformation of the arch domain. In the NVL-bound crystal structure, the arch domain of MTR4 is an open conformation, with the KOW domain clearly separated from the helicase core (Fig. $3 \mathrm{c}$ and Supplementary Fig. 4a). The NVL W-AIM polypeptide chain binds in an extended conformation, similar to that reported for NRDE- $2^{31}$, Nop $53^{36}$ and Air2 ${ }^{34}$ (Supplementary Fig. 4b, 4c). The hydrophobic side chains of Trp $173_{\mathrm{NVL}}, \quad$ Phe $174_{\mathrm{NVL}}$, and

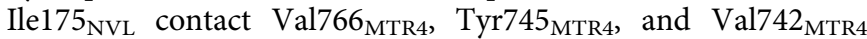
respectively, while Asp $176_{\mathrm{NVL}}$ interacts with Arg743 ${ }_{\text {MTR4 }}$ (Fig. 3d). The NVL polypeptide chain then forms a pronounced bend at Pro179 ${ }_{\mathrm{NVL}}$ (which contacts Phe653 $3_{\mathrm{MTR}}$ ) and continues to form a crystal contact, docking at the Trf4-binding site of a symmetry related helicase core (Supplementary Fig. 5a).
Consistent with the structural data, mutation of Asp $176_{\mathrm{NVL}}$ (D176A mutant) impairs the binding of NVL ${ }^{167-216}$ to MTR4 $\Delta \mathrm{N}$ in a GST pull down experiment (Supplementary Fig. 5b).

Finally, we tested the effect of the MTR4-interacting residues of NVL in human cells. To this end, we carried out coimmunoprecipitation (co-IP) assays in HeLA cells stably expressing MTR4 with an eGFP 'localization and affinity purification' (LAP) tag at the N-terminus and transiently expressing FLAGtagged full-length NVL constructs (wild type, or with the W173A/ I175A mutation or with deletion of the entire hydrophobic segment). Western blotting analysis confirmed that wild type NVL bound MTR4 and that disruption of the 172-180 segment, either by mutation or deletion, impaired the NVL-MTR4 interaction (Fig. 3e, compare lanes 8, 9, 10). Taken together, we conclude that NVL interacts with the KOW domain of MTR4 using a short linear W-AIM sequence. The NVL W-AIM is more hydrophobic than the Nop53 AIM ${ }^{35}$, rationalizing why it binds MTR4 with an order of magnitude higher affinity than the yeast Nop53-Mtr4 $4^{36}$ and human Nop53-MTR4 interactions (Supplementary Fig. 3d).

The unstructured region of ZCCHC8 interacts with the MTR4 KOW domain. Identification of the W-AIM sequence in NVL 
a

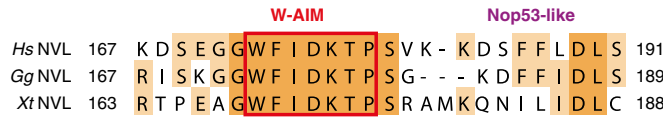

Hs NVL 192 CEKS-NPKKP I T E I QDSKDS SLLESD 216 GgNVL 190 EDGEGDEKKL I S- - EK STEFSVLESE 213 $X$ NVL 189 ED- S-AEVSHSA- - QNPPDLSLLESE 210

C

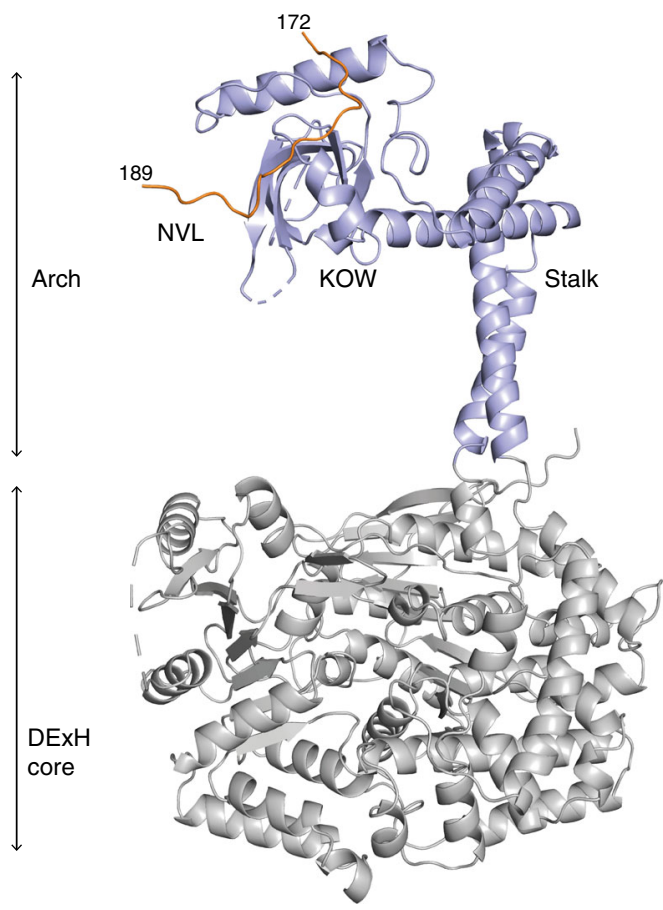

d

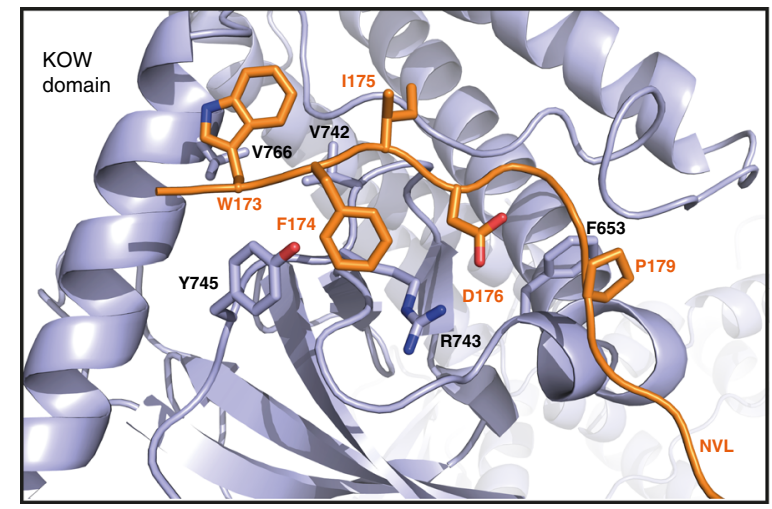

b

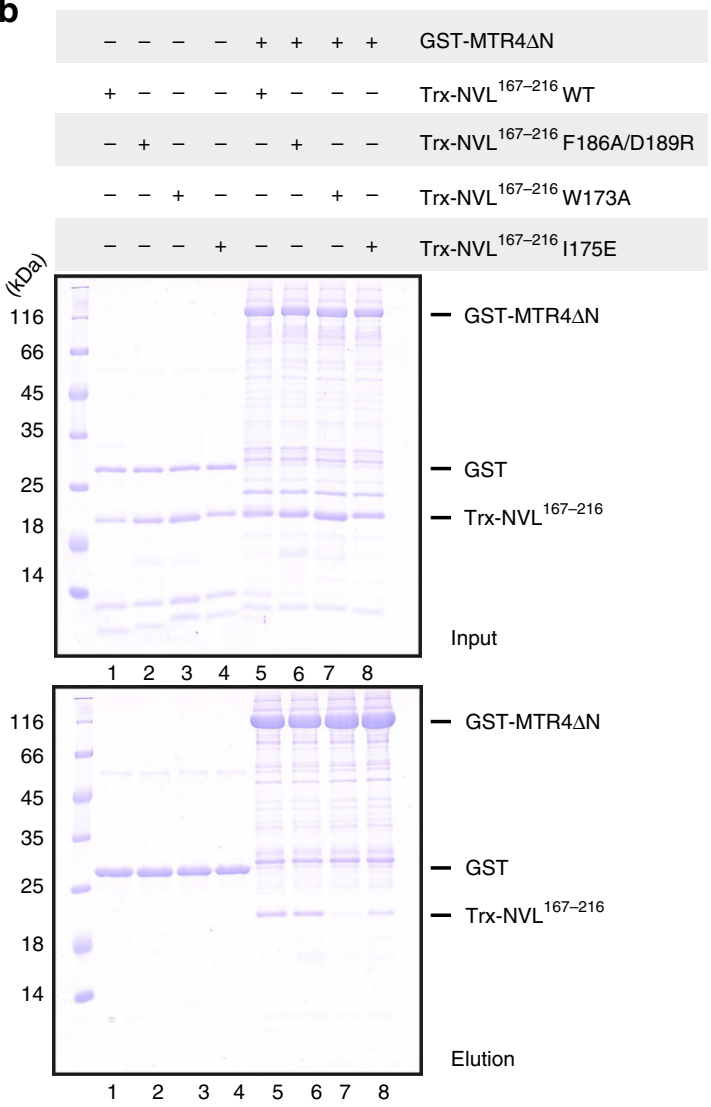

e

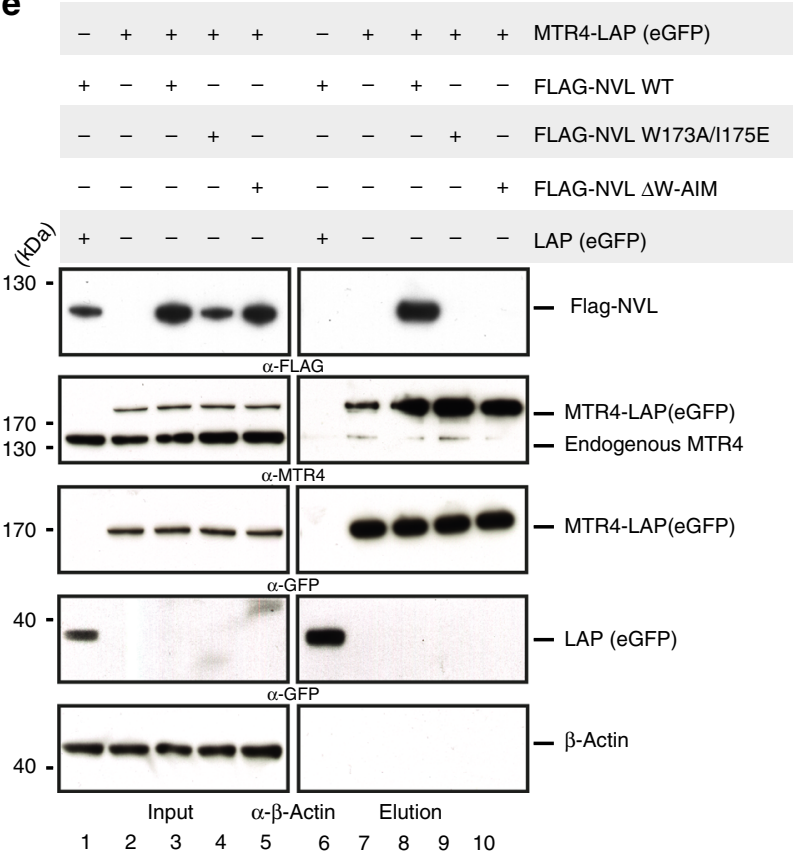

core of MTR4 and activates $\mathrm{it}^{37}$. The N-terminal portion of ZCCHC8 is expected to contain an additional MTR4-binding site $^{37}$. Within the N-terminal portion, we focused our attention on the linker between the coiled-coil and the Zinc-knuckle domains, as it appeared to contain an NVL-like tryptophancontaining sequence (Supplementary Fig. 6a). We expressed a large portion of this linker (residues 91-211) tagged to maltose binding protein (MBP-ZCCHC8 ${ }^{91-211}$ ) and purified the resulting 
Fig. 3 The vertebrate specific W-AIM in NVL is crucial for binding to MTR4 KOW. a Sequence alignment of vertebrate specific insertion regions of representative NVL sequences, Homo sapiens $(H s)$, Gallus gallus $(\mathrm{Gg})$, Xenopus tropicalis (Xt), highlighting the W-AIM (GWFIDKTP) (red box), and the Nop53-like region (LFXQD) (purple). The sequences were obtained from the UniProt database and aligned using the T-coffee server ${ }^{59}$. b Protein coprecipitations by pull down assays. GST-tagged MTR4 $\Delta$ N was incubated with either wild type Trx-NVL ${ }^{167-216}$ or its indicated mutant before coprecipitation with glutathione sepharose beads. A total of 3\% of the input (top) and 30\% of the eluates (bottom) were analyzed on 15\% SDS-PAGE gels and visualized by staining with coomassie brilliant blue. c Overall structure of MTR4 $\Delta \mathrm{N}_{2}{ }^{70-1042}-\mathrm{NVL} \mathrm{L}^{167-216}$ complex, with the DExH core of MTR4 colored in gray and arch colored in light blue. NVL is colored in orange. $\mathbf{d}$ Zoom-in view of the interactions between MTR4 KOW (light blue) domain and NVL (orange). Domains are colored as in Fig. $3 a$ and viewed are $90^{\circ}$ rotation along the horizontal and vertical axes with respect to the view in Fig. 3 a. Residues discussed in the text are highlighted and labeled. e Cellular co-IP assay. FLAG-tagged wild type NVL construct, or its indicated mutant variant, were transiently expressed in cells stably expressing MTR4-LAP. After precipitation of MTR4-LAP, a total of $0.5 \%$ of the input (left) and $8.0 \%$ of the eluates (right) were analyzed on 4-12\% SDS-PAGE gel followed by western blotting analysis. The primary antibody used is indicated below the panel

\begin{tabular}{|c|c|}
\hline & Mtr4 $\Delta N^{70-1042}-N^{167-216}$ \\
\hline \multicolumn{2}{|l|}{ Data collection } \\
\hline Space group & $P 6_{1}$ \\
\hline \multicolumn{2}{|l|}{ Cell dimensions } \\
\hline$a, b, c(\AA)$ & $184.37,184.37,90.53$ \\
\hline$\alpha, \beta, \gamma\left({ }^{\circ}\right)$ & $90.0,90.0,120.0$ \\
\hline Resolution $(\AA)$ & $92.18-3.07$ \\
\hline$R_{\text {sym }}$ or $R_{\text {merge }}$ & $15.7(527.6)^{a}$ \\
\hline$|/ \sigma|$ & $12.6(0.9)$ \\
\hline Completeness (\%) & $99.9(99.5)$ \\
\hline Redundancy & 16.5 \\
\hline $\mathrm{CC}_{1 / 2}$ & $99.9(62.7)$ \\
\hline \multicolumn{2}{|l|}{ Refinement } \\
\hline Resolution $(\AA)$ & $60.35-3.07(3.22-3.07)$ \\
\hline No. reflections & 32,919 \\
\hline$R_{\text {work }} / R_{\text {free }}$ & $22.3 / 25.7$ \\
\hline \multicolumn{2}{|l|}{ No. atoms } \\
\hline Protein & 7074 \\
\hline Ligand/ion & 58 \\
\hline Water & 6 \\
\hline \multicolumn{2}{|l|}{$B$-factors } \\
\hline Protein & 123.57 \\
\hline Ligand/ion & 138.03 \\
\hline Water & 92.34 \\
\hline \multicolumn{2}{|l|}{ R.m.s. deviations } \\
\hline Bond lengths $(\AA)$ & 0.003 \\
\hline Bond angles $\left({ }^{\circ}\right)$ & 0.478 \\
\hline
\end{tabular}

${ }^{a}$ values in parentheses are for highest-resolution shell

construct for pull-down assays with the versions of GST-tagged MTR4 described above (Fig. 1a). MBP-ZCCHC891-211 coprecipitated with GST-MTR4- $\Delta$ N but not with GST-MTR4$\Delta \mathrm{N} \Delta$ arch (Fig. $4 \mathrm{~b}$, lanes 6 and 7 ). Similar to the results we had obtained for NVL ${ }^{167-216}$, the pull-down assays showed that MBPZCCHC8 ${ }^{91-211}$ interacts with GST-MTR4-KOW (Fig. 4b, lane 8). Next, we determined the affinity of the MTR4-KOWZCCHC8 ${ }^{91-211}$ interaction using microscale thermophoresis (MST) with a fluorescent-tagged version of $\mathrm{ZCCHC} 8^{91-211}$ (that we had engineered by fusing a YFP to the C-terminus). In this quantitative assay, we measured a dissociation constant $\left(K_{\mathrm{D}}\right)$ of $\sim 0.3 \mu \mathrm{M}$ (Fig. 4c), indicating a higher affinity than that of the NVL ${ }^{167-216}$ fragment. To corroborate these results, we pre-formed a GST-MTR4- $\Delta \mathrm{N}$ - Trx-NVL ${ }^{167-216}$ complex and incubated it with increasing amounts of MBP-ZCCHC $8^{91-211}$ before subjecting the mixtures to GST pull-down assays. The competition assay showed that ZCCHC8 ${ }^{91-211}$ could displace NVL ${ }^{167-216}$ from the pre-formed complex (Fig. 4d, lanes 5-8), suggesting that they interact with the exosome helicase in a mutually exclusive manner.

Identification of C-AIM: a cysteine arch-interacting motif of ZCCHC8. We proceeded to identify the MTR4-binding site within the $\mathrm{N}$-terminal region of $\mathrm{ZCCHC}$. We first interrogated the NVL-like tryptophan-containing patch (GWEIPK, residues 197-202, Fig. 5a). Surprisingly, mutation of Trp198 and Lys202 (W198A/K202E mutant) did not alter the interaction of ZCCHC8 ${ }^{91-211}$ with MTR4- $\Delta \mathrm{N}$ in GST pull down assays (Fig. 5b, lane 7), indicating that this patch of $\mathrm{ZCCHC} 8$ is not a bona-fide W-AIM sequence.

To identify the arch-interacting motif in $\mathrm{ZCCHC} 8^{91-211}$, we took a similar bioinformatics approach as described above for NVL. When analyzing the evolutionary conservation of ZCCHC8 ${ }^{91-211}$, we identified a conserved patch centered at the FCLDKLG segment (residues 178-184, Fig. 5a). Mutation of Phe178 and Asp181 (F178A/D181R mutant) in this segment impaired the interaction with MTR4- $\Delta \mathrm{N}$ (Fig. 5b, lane 6). This cysteine-centered arch-interacting motif (C-AIM) is loosely related to the Nop53 AIM and the NVL W-AIM segments, and is thus expected to bind to the same site of the KOW domain. Consistently, the Arg743 reverse-charge substitution (R743E) in MTR4 also impaired the binding of ZCCHC8 ${ }^{91-211}$ (Fig. 5c, lane 8).

ZCCHC8 also contains a non-canonical arch-interacting motif: I-AIM. While attempting to narrow down the KOW binding region of $\mathrm{ZCCHC} 8$ further, we observed that $\mathrm{N}$-terminal truncation of $\mathrm{ZCCHC} 8^{91-211}$ resulted in a near loss of MTR4 binding (Supplementary Fig. 6b). Based on these results, we reasoned that ZCCHC8 ${ }^{91-211}$ might harbor an additional MTR4-binding motif that would be predicted to bind to an adjacent surface on the KOW domain (and thus show no resemblance to the previously identified arch-interacting motifs). Using bioinformatics approaches, we identified another conserved segment upstream of C-AIM (IEEF, residues 112-115). Indeed, mutation of Ile112 and Phe115 (I112R/F115R mutant) severely weakened the MTR4- $\Delta$ N - ZCCHC8 ${ }^{91-211}$ interaction in GST pull-down assays (Fig. 5b, lane 8). Thus, this segment (which we refer to as an isoleucinecentered arch-interacting motif, or I-AIM) also contributes to MTR4 binding. Next, we mapped the possible MTR4-binding site of I-AIM. The $\beta$-barrel of the MTR4 KOW domain is structurally related to that of Tudor domains (Supplementary Fig. 7a), small globular folds that generally present a hydrophobic pocket for binding methylated arginines and lysines ${ }^{44}$. Although there is little overall sequence similarity, MTR4-KOW features hydrophobic residues at the equivalent position of the substrate-binding residues of Tudor domains (Supplementary Fig. $7 b)^{44,45}$. In particular, MTR4 Phe677 is evolutionarily conserved (Supplementary Fig. 7b) and is located on a surface adjacent to the Arg743 site where the Nop53 like AIM, W-AIM and C-AIM sequences are recognized (Supplementary Fig. 7c). In line with this site being used for the additional I-AIM sequence of ZCCHC8 ${ }^{91-211}$, mutation of Phe677 (F677E) disrupted binding of ZCCHC8 ${ }^{91-211}$ (Fig. 5c, lane 7) and did not affect the binding 
a

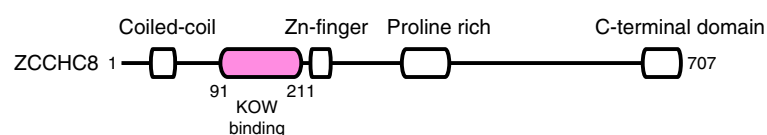

b

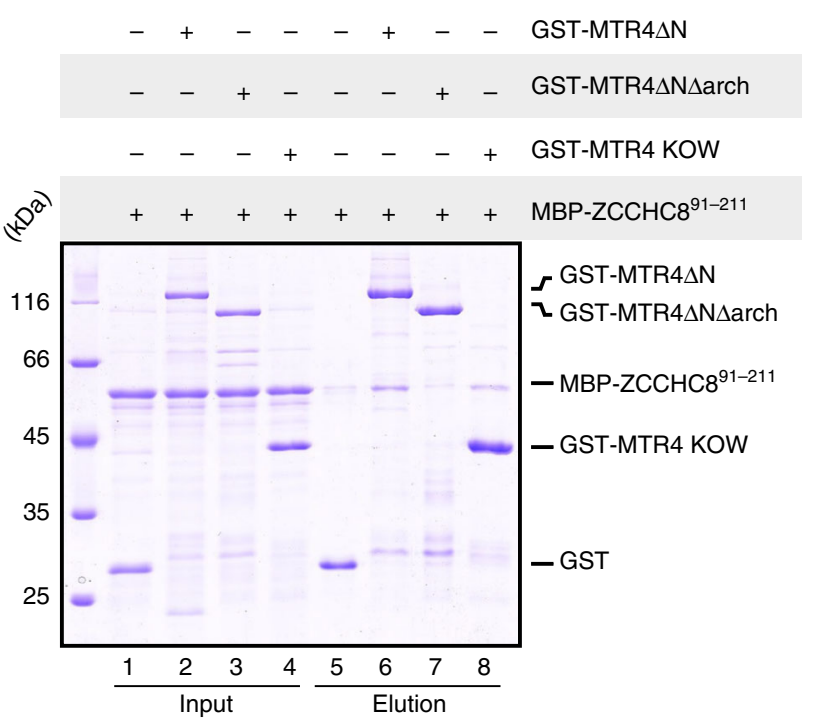

C

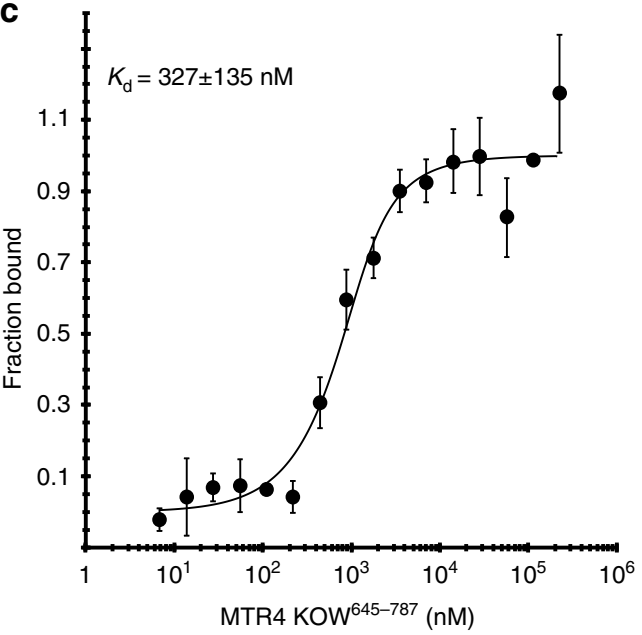

d

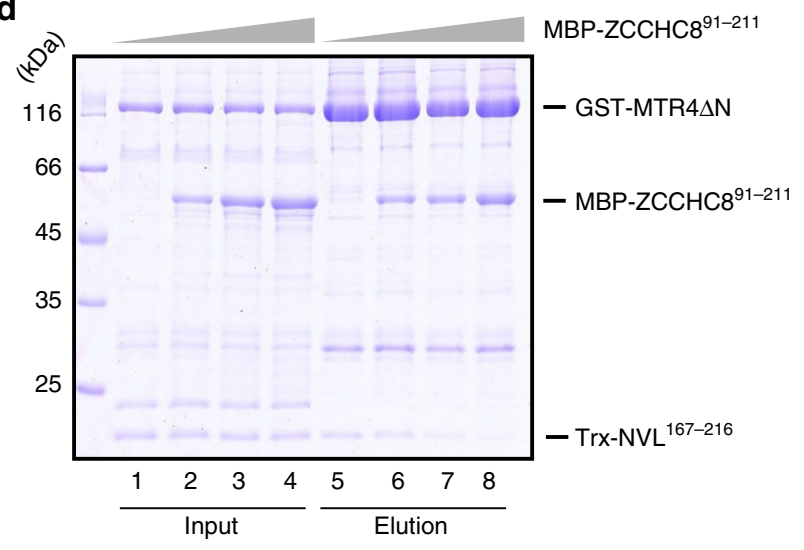

of NVL ${ }^{167-216}$ (Fig. 2d, lane 7) or human Nop53 (Supplementary Fig. 3c, lane 7).

We tested the importance of the I-AIM segment in human cells. HeLa LAP-MTR4 cells were transfected with FLAG-tagged full-length ZCCHC8 constructs (wild type, the I-AIM I112R/
Fig. 4 The N-terminus of ZCCHC8 interacts with the MTR4 KOW domain. a Schematic representation of the domain organization of $\mathrm{ZCCHC}$. Domain boundaries are obtained from previous studies 37,43 and computational sequence analysis. b Protein co-precipitations by pull down assays. GST-tagged MTR4 $\Delta \mathrm{N}, \mathrm{MTR} 4 \Delta \mathrm{N} \Delta$ arch, and MTR4 KOW were incubated with MBP-ZCCHC8 $91-211$ before co-precipitation with glutathione sepharose beads. A total of $3 \%$ of the input (lanes 1-4) and $30 \%$ of the eluates (lanes 5-8) were analyzed on 12\% SDS-PAGE gels and visualized by staining with coomassie brilliant blue. c Microscale thermophoresis experiment with MTR4 KOW and MBP-ZCCHC8 91-211_

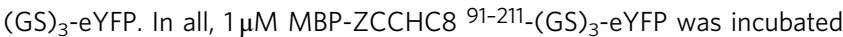
with increasing concentrations of MTR4 KOW and thermophoresis was measured by tracking the fluorescence of the ZCCHC8-YFP fusion protein. The binding curve was calculated using $\mathrm{MO}$ software (Nanotemper technologies) and the dissociation constant $\left(K_{d}\right)$ is given in the inset. The error bars represent the standard deviations of each data point calculated from three independent thermophoresis measurements. d Competition experiment using GST pull down assay technology. A preformed GSTMTR4 $\Delta \mathrm{N}$ :Trx-NVL ${ }^{167-216}$ complex was incubated with increasing concentrations of MBP-ZCCHC8 ${ }^{(91-211)}$ before precipitation with glutathione sepharose beads. A total of $3 \%$ of the input (lanes, 1-4) and $30 \%$ of the eluates (5-8) were analyzed on 15\% SDS-PAGE gels and visualized by staining with coomassie brilliant blue

F115R double mutant or a deletion construct lacking the ZCCHC8 CTD). Western blotting analysis of the resulting coIPs revealed that disrupting the I-motif by mutation is sufficient to impair the ZCCHC8-MTR4 interaction, whereas deletion of the CTD did not significantly affect complex formation (Fig. 5d, lanes 8, 9, 10). These mutations also did not affect the ZCCHC8RBM7 interaction (Supplementary Fig. 6c). The CTD of ZCCHC8 has been shown to bind the DExH core of MTR4 and to enhance helicase activity ${ }^{37}$. In contrast, we found that neither the ZCCHC8 nor the NVL AIM motifs have a significant effect on the catalytic properties of MTR4, as judged by assaying both ATP hydrolysis and RNA helicase activities (Supplementary Fig. 8). These results suggest a division of labor of the N-terminal and Cterminal ZCCHC8 regions: while the C-terminal domain regulates the activity of the helicas $\mathrm{e}^{37}$, the $\mathrm{N}$-terminal region plays has a central scaffolding role in incorporating MTR4 into the NEXT complex.

\section{Discussion}

In this study, we show that the human nuclear exosome adaptors NVL and ZCCHC8 bind the MTR4 KOW domain on a surface that is also employed by Nop $53^{36}$ and NRDE- $2^{31}$ using distinct arch-interacting motifs ${ }^{35}$. The AIMs of Nop53/Utp18/Air2 $\left(\mathrm{LFx} \phi \mathrm{D}(\mathrm{x})_{1-2} \mathrm{G} / \mathrm{P}\right), \quad \mathrm{NVL}$ (GWFIDKTP), ZCCHC8 (NFCLDKLG), and NRDE-2 (SFRTDKKP) can best be considered as subfamilies of canonical AIMs. With insight, sequences in both NVL and ZCCHC8 that at first glance appeared to resemble known AIMs instead contain individual amino acids that are likely to prevent MTR4 binding. The consensus sequence of canonical bona-fide AIMs can thus be re-defined as $\operatorname{x\omega xxD}(x)_{1 /}$ ${ }_{2} \mathrm{G} / \mathrm{P}$, with a C-terminal glycine or proline residue that allows the polypeptide chain to bend as it extends away from its binding site. The aromatic $(\omega)$ and polar/non-polar amino acids might vary, but tend to be conserved within individual subfamilies of canonical AIMs. Despite the variability, all canonical AIM sequences are recognized, in a mutually exclusive manner, at a surface pocket of the MTR4 KOW domain that is defined by the presence of Arg743 (yeast Mtr4 Arg774). Mutation of this surface pocket may thus be a useful tool to probe new MTR4-interacting 
a

Hs ZCCHC8 91 DGP I LQ I LFMNNA I SKQYHQE I EEFV SNLVKR 122

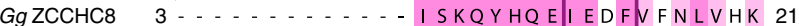

XIZCCHC8 78 DGPLLQILFMNHII SKQYHQELIEEFITSLFQK 109

Hs ZCCHC8 123 FEEQQKNDVEKT SFNLLPQPS S I VLEEDHKVE 154 GgZCCHC8 22 YEDQQRDEQEKTHFSLKPQP S SVVLEEDYKTA 53 XIZCCHC8 110 YEEHRKSNSEKT SFN IKPQPS S I LLEENDDS - 140

HS ZCCHC8 155 ESCA I KNNKEAFSVVGSVLYFTNFCLDKLGQP 186 Gg ZCCHC8 54 S SNSVKK I KEAFSVVGSVLYFTNFCLDKLGQP 85 XIZCCHC8 141 DADTMKN IKQAF SVVGSVQYFKNFCLDKLGQP 172

Hs ZCCHC8 187 LLNENPQLSEGWEIPKYHQVFSH C-AIM

GgZCCHC8 86 ILNENPQLTEGWEIPKYOOVFSO IL

XIZCCHC8 173 LLNENPQLTEGWDIPKYQQVFTQIV NVL like

b
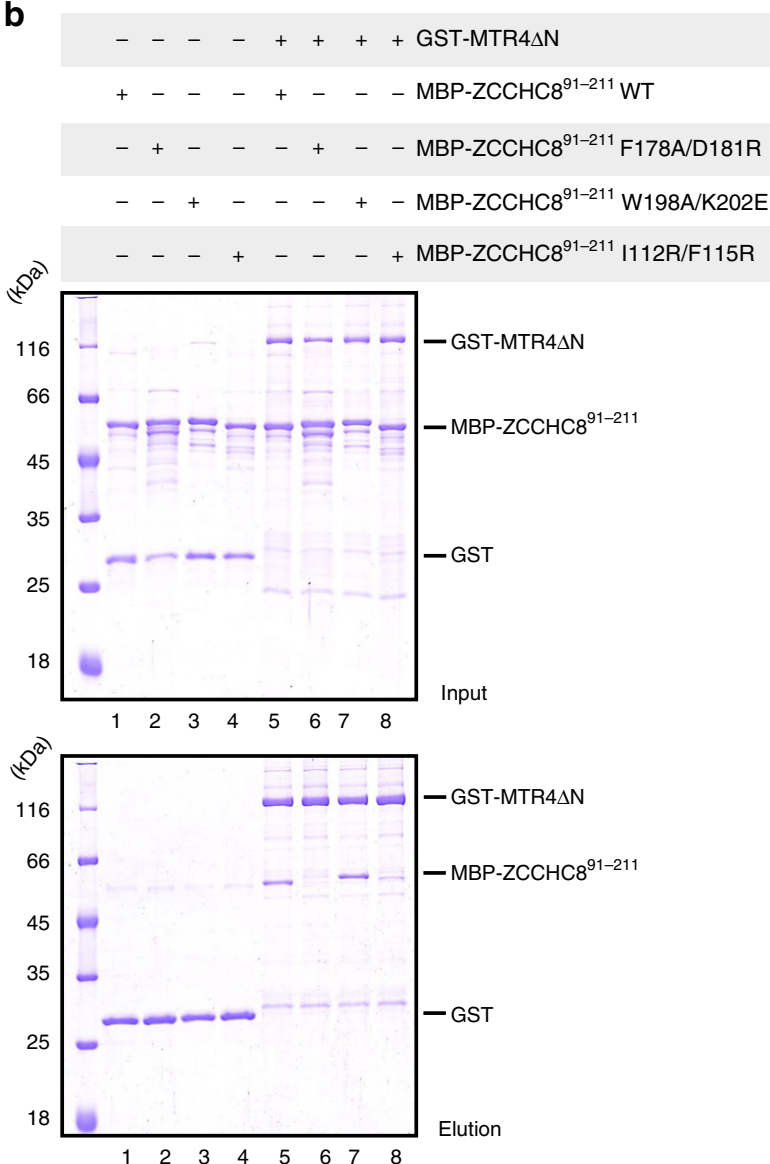

C

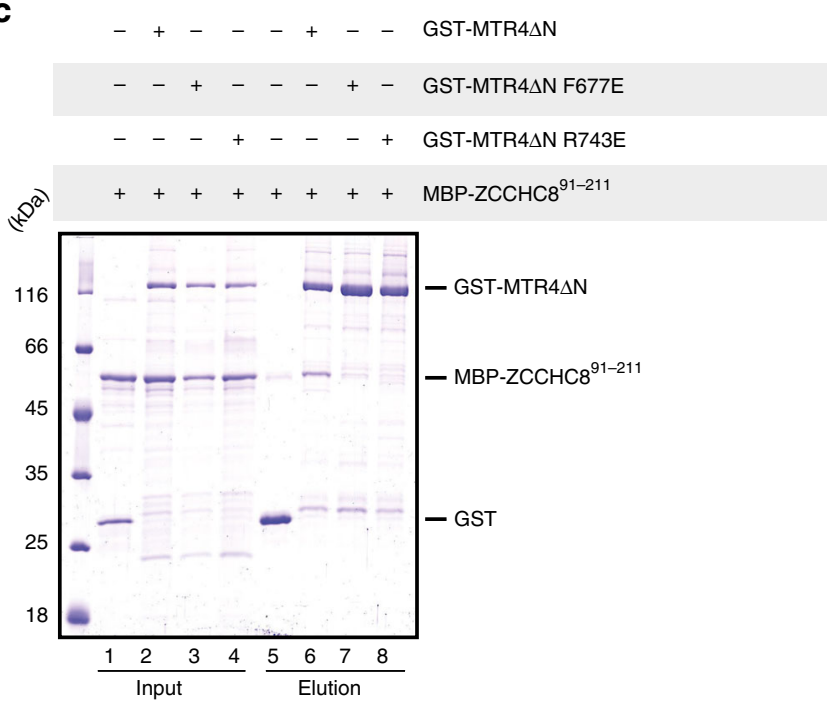

d

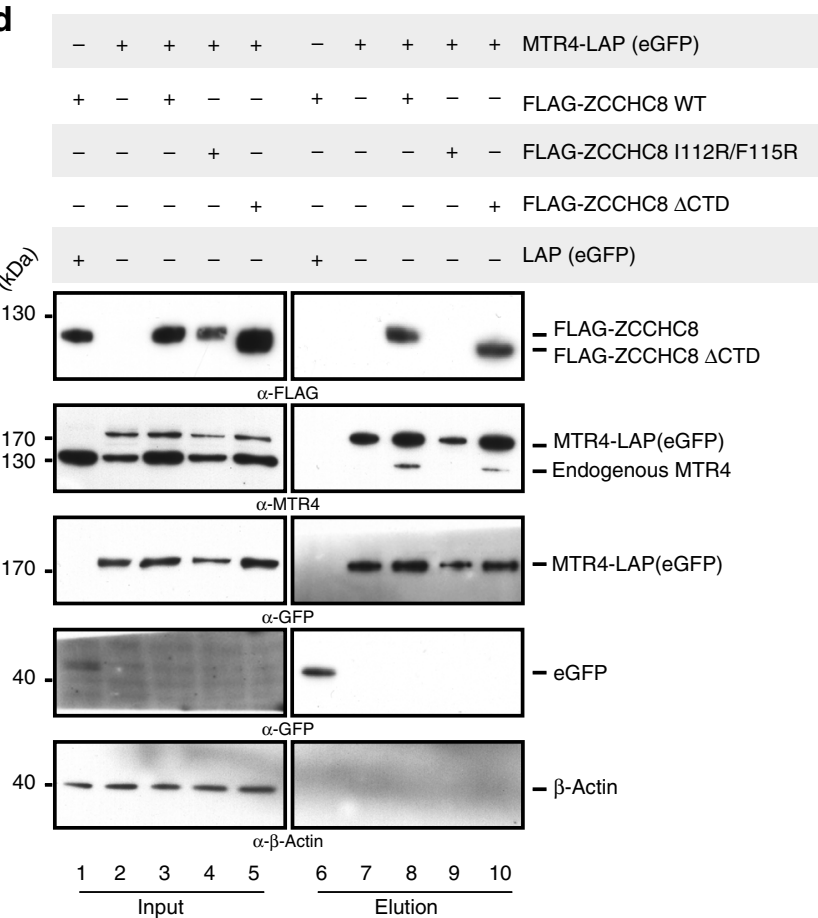

Fig. 5 Analysis of the ZCCHC8-MTR4 KOW complex by site directed mutagenesis. a Sequence alignment of the region between the predicted coiled coil domain and the zinc finger of ZCCHC8 from representative metazoan species, Homo sapiens (Hs), Gallus gallus (Gg), Xenopus laevis (XI), highlighting NVLlike region (red), Nop53-like AIM (C-AIM) and ZCCHC8 specific I-AIM (purple boxes). The sequences were obtained from the UniProt database and aligned using the T-coffee server ${ }^{59}$. b Protein co-precipitations by pull down assays testing ZCCHC8 ${ }^{91-211}$ mutants for MTR4 4 N binding ability. GSTtagged MTR $4 \Delta \mathrm{N}$ was incubated with either ZCCHC $8^{91-211}$ WT or mutants before co-precipitation with glutathione sepharose beads. A total of $3 \%$ of the input (top) and $30 \%$ of the eluates (bottom) were analyzed on $12 \%$ SDS-PAGE gels and visualized by staining with coomassie brilliant blue. c Protein coprecipitations by pull down assays testing MTR4 $\mathrm{N}$ mutants for their ZCCHC8 ${ }^{91-211}$ binding ability. GST-tagged MTR4 $\mathrm{N}$ (WT or mutant variants) were incubated with MBP-ZCCHC8 ${ }^{91-211}$ before co-precipitation with glutathione sepharose beads. A total of $3 \%$ of the input (lanes, 1-4) and 30\% of the eluates (5-8) were analyzed on 12\% SDS-PAGE gels and visualized by staining with coomassie brilliant blue. d Cellular co-IP assay. FLAG-tagged ZCCHC8 constructs (WT/IF mutant/CTD deletion) were transiently expressed in cells stably expressing MTR4-LAP. After precipitation of MTR4 taking advantage of the LAP tag, a total of $0.5 \%$ of the input (left) and $8.0 \%$ of the eluates (right) were analyzed on $4-12 \%$ SDS-PAGE gel followed by western blotting analysis. The primary antibody used is indicated below the panel

proteins that contain arch-interacting motifs, as these motifs are difficult to identify due to the degeneracy of their consensus sequence. Furthermore, we found that ZCCHC8 harbors an additional arch-interacting motif that does not conform to the canonical AIM consensus sequences. These findings show how different exosome adaptor proteins have evolved similar mechanisms to recognize MTR4 in a specific and mutually exclusive manner, but can also modulate the affinity and thus selectivity with which they are ultimately recruited to the nuclear exosome. 


\section{Methods}

Protein expression and purification. Human MTR4 constructs (full-length, MTR4- $\Delta N$ (75-1042), MTR4-KOW (645-787) and MTR4- $\Delta$ N $\Delta$ arch, where residues 598-842 were substituted by 2 xGS linker) were expressed as 6xHis-GSTtagged fusion (cleavable with 3 C protease) proteins in BL21 star (DE3) E. coli cells, grown either in terrific broth (for biochemical studies) or minimal medium supplemented with ${ }^{15} \mathrm{~N}$ labelled ammonium chloride and $/$ or ${ }^{13} \mathrm{C}$ labeled Glucose (for NMR studies). The proteins were purified using a Ni-nitrilotriacetate (NTA) affinity column and a heparin column (GE healthcare) for ion exchange chromatography. When appropriate, the His-GST tag was cleaved at this point by incubation with $3 \mathrm{C}$ protease, followed by removal of the tag with an additional Ni-NTA affinity step. Finally, the protein was subjected to size exclusion chromatography on a Superdex 200 column (GE healthcare) in $50 \mathrm{mM}$ Hepes/ $\mathrm{NaOH}$ pH 7.5, $150 \mathrm{mM} \mathrm{NaCl}, 5 \%$ (v/v) glycerol, $2 \mathrm{mM}$ DTT.

The human MTR4 construct used for crystallization (71-1042) was cloned as GST fusion construct with a TEV cleavage site immediately preceding the MTR4 sequence which when cleaved would leave a glycine (the natural 70th residue in MTR4) yielding MTR4 protein with residues 70-1042 (referred to as MTR $4 \Delta N_{2}$ in the text). The construct was purified as described above.

The primers used for cloning all the constructs described are listed in Supplementary table 1 .

All NVL and ZCCHC8 constructs and truncation mutants described in the text were expressed as $6 \mathrm{xHis}-\mathrm{Trx}$ and $6 \mathrm{xHis-MBP}$ tagged fusion (cleavable with $3 \mathrm{C}$ protease) proteins, respectively in BL21 star (DE3) E. coli cells. The proteins were purified using a Ni-NTA affinity column. When appropriate, the tags were cleaved upon incubation with $3 \mathrm{C}$ protease at this step, followed by an additional Ni-NTA affinity step for the removal of the tag. In case of ZCCHC8, the tag cleavage was performed only in the presence of MTR4 to prevent the protein from precipitating. In the final purification step, the proteins were subjected to size-exclusion chromatography on a Superdex 75 in $50 \mathrm{mM}$ Hepes/ $\mathrm{NaOH}$ pH 7.5, $150 \mathrm{mM} \mathrm{NaCl}$, $2 \mathrm{mM}$ DTT.

Biophysical assays. The microscale thermophoresis measurements were performed on a NanoTemper Monolith NT.115 machine. Before the measurements, all samples were dialyzed against a buffer containing in $50 \mathrm{mM}$ Hepes/ $\mathrm{NaOH} \mathrm{pH}$ $7.5,150 \mathrm{mM} \mathrm{NaCl}, 5 \%(\mathrm{v} / \mathrm{v})$ glycerol, $0.5 \mathrm{mM}$ TCEP. For NVL, $50 \mathrm{nM}$ of Trx$\mathrm{NVL}^{167-217}-(\mathrm{GS})_{3}$-eYFP was incubated with increasing concentrations of unlabeled MTR4 KOW and thermophoresis was measured with an MST power of $20 \%$ and an LED power of $20 \%$. For ZCCHC8, $1 \mu \mathrm{M}$ of MBP-ZCCHC8 ${ }^{11-211}$-(GS) $)_{3}$-eYFP was incubated with increasing concentrations of unlabeled MTR4-KOW and thermophoresis was measured with an MST power of 70\% and an LED power of $15 \%$. In both cases, the MTR4 KOW concentration series was produced by serial dilution (1:1). Titrations were performed in triplicates and the data were analyzed using the Thermophoresis with T-Jump strategy option with the MO software (NanoTemper Technologies).

Isothermal calorimetry experiments were carried out using the ITC200 Isothermal titration calorimeter from Microcal. Before the measurement, all samples were dialysed against a buffer containing $50 \mathrm{mM}$ Hepes/ $\mathrm{NaOH}$ pH 7.5, $150 \mathrm{mM} \mathrm{NaCl}$, and $0.5 \mathrm{mM}$ TCEP. For NVL: MTR4- $\Delta \mathrm{N}$ (the reactant) samples were concentrated to $40 \mu \mathrm{M}$ and NVL (the injectant) to $500 \mu \mathrm{M}$. For human NOP53: MTR4-KOW (the reactant) samples were concentrated to $100 \mu \mathrm{M}$ and NOP53 (the injectant) to $1 \mathrm{mM}$. Titrations were carried out at $25^{\circ} \mathrm{C}$ with $2 \mu \mathrm{L}$ of the injectant per injection added to $200 \mu \mathrm{L}$ of reactant cell solution. All data were processed and curves fitted using Origin5.0.

Biochemical assays. For pull-down assays, appropriate protein mixtures were incubated in $50 \mathrm{mM}$ Hepes (pH 7.5), $150 \mathrm{mM} \mathrm{NaCl}, 5 \%(\mathrm{v} / \mathrm{v})$ glycerol, $0.05 \%(\mathrm{v} / \mathrm{v})$ NP40, $1 \mathrm{mM}$ DTT for $30 \mathrm{~min}$ at $4{ }^{\circ} \mathrm{C}$. For ZCCHC8, $2 \mu \mathrm{M}$ GST-MTR $4 \Delta \mathrm{N}$ was incubated with $8 \mu \mathrm{M}$ MBP-ZCCHC8 constructs. For NVL, $10 \mu \mathrm{M}$ GST-MTR $4 \Delta \mathrm{N}$ was incubated with $20 \mu \mathrm{M}$ Trx-NVL constructs. For NOP53, $30 \mu \mathrm{M}$ GST-

MTR $4 \Delta \mathrm{N}$ was incubated with $60 \mu \mathrm{M}$ Trx-NOP53 $3^{84-123}$ in a total volume of $30 \mu \mathrm{l}$. The protein mixtures were then incubated with Glutathione sepharose beads (GE healthcare) for $2 \mathrm{~h}$. Post incubation, the beads were washed three times with $0.1 \mathrm{ml}$ incubation buffer and the retained material was eluted with incubation buffer supplemented with $30 \mathrm{mM}$ reduced glutathione. Input material (1-3\%) and eluates $(\sim 30 \%)$ were analyzed by SDS-PAGE and Coomassie staining.

For ATPase assays, 150 pmol of MTR $4 \Delta \mathrm{N}$ or MTR $4 \Delta \mathrm{N}$-containing complexes were incubated $40 \mathrm{nmol}$ MESG (2-amino-6-mercapto-7-methylpurine ribonucleoside) and $0.5 \mathrm{U}$ purine nucleoside phosphorylase (Enzchek Phosphate Assay kit, Invitrogen) in a buffer containing $50 \mathrm{mM}$ MOPS pH 6.5, $50 \mathrm{mM} \mathrm{Nacl}$, $2.5 \mathrm{mM} \mathrm{MgCl}_{2}, 5 \mathrm{mM} \beta$-mercaptoethanol and $5 \%(\mathrm{v} / \mathrm{v})$ glycerol. For reactions containing RNA, the mixture was incubated with $2 \mu \mathrm{g}$ poly-U RNA (Sigma). The reaction was initiated by addition of ATP to a final concentration of $1 \mathrm{mM}$. The generation of 2-amino-6-mercapto-7-methylpurine from MESG was monitored by measuring absorbance increase at $360 \mathrm{~nm}$ on a plate reader (Infinite M1000 Pro, Tecan) for $12 \mathrm{~min}$ at $60 \mathrm{~s}$ intervals. The data were normalized by subtracting the $y$ intercept from the raw data. The experiment was performed in triplicate. The mean $(n=3)$ and standard deviation (error bars) were plotted using Graphpad prism 8.

Helicase assays were performed essentially as described by Puno et al. ${ }^{37}$. A duplex RNA was formed by mixing a 5' Fluorescein amidite (FAM) labeled RNA
(FAM-AGCACCGUAAAGACGC) with 1.5 molar excess of complementary RNA with a 25 A overhang (GCGUCUUUACGGUGCUAAAAAAAAAAAAAAAAA AAAAAAAA) in a buffer containing $20 \mathrm{mM}$ Tris- $\mathrm{HCl} \mathrm{pH} 7.5$ and $50 \mathrm{mM} \mathrm{NaCl}$ To anneal the RNA duplex, the mixture was heated to $95^{\circ} \mathrm{C}$ and allowed to cool down slowly to ambient temperature by turning off the heat block. 0.3 pmol of duplex RNA was incubated with $3.75,7.5,30$ pmol of MTR4 $\Delta \mathrm{N}$ or MTR4 $\Delta \mathrm{N}$ containing complexes in a buffer containing $50 \mathrm{mM}$ MOPS pH $6.5,50 \mathrm{mM} \mathrm{Nacl}$, $0.5 \mathrm{mM} \mathrm{MgCl} 2,5 \mathrm{mM} \beta$-mercaptoethanol and $5 \%(\mathrm{v} / \mathrm{v})$ glycerol for $5 \mathrm{~min}$ at $30^{\circ} \mathrm{C}$ The reaction was then initiated by the addition of ATP, $\mathrm{MgCl}_{2}$ and a trap DNA oligo complementary to the FAM labeled RNA (GCGTCTTTACGGTGCT) to a final concentration of $2 \mathrm{mM}, 2 \mathrm{mM}$ and $400 \mathrm{nM}$ respectively. The reactions were quenched after $40 \mathrm{~min}$ by placing the tubes on ice and adding quenching buffer to a final concentration of $0.5 \%(\mathrm{w} / \mathrm{v})$ SDS, $10 \mathrm{mM}$ EDTA, $10 \%(\mathrm{v} / \mathrm{v})$ glycerol and $0.005 \%(\mathrm{w} / \mathrm{v})$ xylene cyanol. The sample were analyzed by electrophoresis on a $15 \%$ acrylamide-Tris base, boric acid, EDTA (TBE) gel. The fluorescence was imaged using a Typhoon FLA 7000. The oligonucleotides used in the assay were obtained from Ella Biotech GmBH.

NMR spectroscopy. NMR measurements of MTR4-KOW were performed in phosphate buffered saline ( $\left.10 \mathrm{mM} \mathrm{PO}_{4}{ }^{3-}, 137 \mathrm{mM} \mathrm{NaCl}, 2.7 \mathrm{mM} \mathrm{KCl}\right)$ mixed with $10 \%(\mathrm{v} / \mathrm{v}) \mathrm{D}_{2} \mathrm{O}$. Backbone chemical shift assignments of the KOW region were obtained from two ${ }^{13} \mathrm{C},{ }^{15} \mathrm{~N}$-labelled samples with protein concentration of $700 \mu \mathrm{M}$ and $500 \mu \mathrm{M}$, respectively. $\mathrm{HNCA}, \mathrm{HNCACB}, \mathrm{HNcoCA}, \mathrm{HNCO}, \mathrm{HNcaCO}$, and $3 \mathrm{D}$ ${ }^{15} \mathrm{~N}$-edited NOESY spectra ${ }^{46}$ were acquired at $298 \mathrm{~K}$ on Bruker Avance III spectrometers at field strengths corresponding to 600 and 800 proton Larmor frequency, equipped with TCI cryogenic probe heads. The ${ }^{15} \mathrm{~N}$ steady-state heteronuclear $\left\{{ }^{1} \mathrm{H}\right\}^{-15} \mathrm{~N}$ NOE experiment was performed at $170 \mu \mathrm{M}$ and a field strength of $500 \mathrm{MHz}$ as described previously ${ }^{47}$. Protein binding was measured from HSQC experiments containing water-flip-back/WATERGATE ${ }^{48,49}$ sequences. Titrations with the NVL peptide were carried out at $298 \mathrm{~K}$ with a KOW concentration of $53 \mu \mathrm{M}$ and in presence of $0.25,0.5,1,2$, and 6 stoichiometric molar equivalents of NVL. Spectra were recorded and processed with Topspin 3.5 and analyzed with CCPNMR Analysis $2.4^{50}$ and Sparky (http://www.cgl.ucsf.edu/ home/sparky). The chemical shift perturbations were calculated as CSP $(\mathrm{ppm})=[6$ $\left.(\Delta \mathrm{H})^{2}+(\Delta \mathrm{N})^{2}\right]^{0.5}$

Crystallization and structure determination. MTR $4 \Delta \mathrm{N}_{2}$ (70-1042) was mixed with 2 molar excess of NVL (167-216) in a buffer containing $20 \mathrm{mM}$ Tris $\mathrm{pH} 7.5$, $150 \mathrm{mM} \mathrm{Nacl}, 1 \mathrm{mM} \mathrm{Mgcl} 2,2 \mathrm{mM}$ ADP and $1 \mathrm{mM}$ TCEP. The crystallization trials were performed using a vapour diffusion setup. Initial crystals were obtained in the A4 (2 M ammonium sulfate, $0.1 \mathrm{M}$ Tris $\mathrm{pH} 8.5)$ condition of SG1 ${ }^{\mathrm{TM}}$ screen (Molecular dimensions). The best diffracting crystals were obtained at a concentration of $10 \mathrm{mg} / \mathrm{ml}$ at $277 \mathrm{~K}$ in $0.1 \mathrm{M}$ Tris $\mathrm{pH} 8,1.8 \mathrm{M}$ ammonium sulfate. The crystals were cryo-protected with reservoir solution supplemented with $30 \%$ glycerol prior flash-freezing in liquid nitrogen. Data were collected at $100 \mathrm{~K}$ at PXIII beamline of the Swiss light Source (Villigen, Switzerland). Data processing and scaling was performed using Xia2/DIALS ${ }^{51,52}$ within CCP4i2 software suite ${ }^{53}$. The crystals belong to the hexagonal spacegroup $169\left(\mathrm{P}_{1}\right)$ containing one molecule (MTR4 $\Delta \mathrm{N}_{2}-\mathrm{NVL}^{167-216}$ ) in the asymmetric unit and diffract to $3.07 \AA$ A resolution The structure was solved by molecular replacement with Phaser ${ }^{54}$ within Phenix using the co-ordinates of the DExH core (98-593 and 847-1042) and KOW domain (645-787) of MTR4 (PDB 6IEH) ${ }^{31}$ as search models. The model was manually completed in $\operatorname{COOT}^{55}$ and refined using phenix.refine ${ }^{56}$. The optimal TLS groups for TLS refinement were determined using TLSMD server ${ }^{57} .96 .1 \%$ of the protein backbone dihedral angles in the final model are in Ramachandran favored region. The figures of crystallographic models were prepared using pyMOL (Schrödinger, LLC).

Cell culture and co-immunoprecipitation assays. Human HeLa Kyoto LAP, MTR4-LAP, RBM7-LAP cell lines were generated as outlined by Poser et al. ${ }^{58}$ and used for example by Lubas et al. ${ }^{27}$ (RBM7-LAP) and Meola et al. ${ }^{30}$ (MTR4-LAP) Briefly, HeLa Kyoto LAP MTR4-LAP, RBM7-LAP cells were cultured in DMEM + $10 \%$ FBS. Transfection was carried out using $10 \mu \mathrm{g}$ of plasmid containing FLAGtagged NVL or ZCCHC8 constructs, with Lipofectamine 2000 (Thermo Fisher) following the manufacturer's instructions. Forty-eight hours after transfection the cells were collected and resuspended in extraction buffer $(150 \mathrm{mM} \mathrm{NaCl}, 20 \mathrm{mM}$ HEPES pH 7.4, 0.5\% (v/v) Triton X-100) containing Protease Inhibitors (Roche). The lysates were sonicated twice for $5 \mathrm{~s}$ at $20 \mathrm{~W}$, and cell debris was removed by centrifugation at $10,000 \mathrm{~g}$ for $10 \mathrm{~min}$. Lysates were incubated $1 \mathrm{~h}$ with Dynabeads M-270 Epoxy (Invitrogen) coupled to a polyclonal llama anti-GFP antibody. Beads were washed three times in extraction buffer, then incubated $20 \mathrm{~min}$ at $25^{\circ} \mathrm{C}$ with 100 units of Benzonase (Sigma) and $2 \mathrm{mM} \mathrm{MgCl}_{2}$. Beads were then washed twice in extraction buffer and proteins were eluted using NuPage LDS Sample Buffer (Invitrogen) at $70^{\circ} \mathrm{C}$ for $10 \mathrm{~min}$, and NuPage Sample Reducing Agent was added. The input material $(0.5 \%)$ and the eluate $(8.0 \%)$ were analyzed by SDS-PAGE on a NuPage Novex $4-12 \%$ Bis-Tris gel (Invitrogen). Western blotting analysis was performed following standard protocols. Following primary antibodies were used: anti-FLAG M2 (Dilution 1:50000; Sigma, F1804), anti-MTR4 (Dilution 1:4000; Abcam, ab70551), anti-GFP (Dilution 1:1000; Santa Cruz Biotechnology, SC-9996), 
anti- $\beta$-actin (Dilution 1:100000; Sigma, A2228) and anti-RBM7 (Dilution 1:1000; human protein atlas, HPA013993). Anti-mouse and anti-rabbit secondary antibodies coupled to Horseradish Peroxidase (Dako) were used.

Reporting summary. Further information on research design is available in the Nature Research Reporting Summary linked to this article.

\section{Data availability}

A reporting summary for this Article is available as a Supplementary Information file NMR backbone chemical shifts of the human MTR4 KOW domain were deposited at the BMRB under accession number 27831. The coordinates and the structure factors have been deposited in the Protein Data Bank with accession code PDB ID 6RO1. The source data are provided in Supplementary Fig. 10. All data is available from the corresponding author upon reasonable request.

Received: 14 January 2019 Accepted: 7 July 2019

Published online: 29 July 2019

\section{References}

1. Chlebowski, A., Lubas, M., Jensen, T. H. \& Dziembowski, A. RNA decay machines: the exosome. Biochim Biophys. Acta 1829, 552-560 (2013).

2. Zinder, J. C. \& Lima, C. D. Targeting RNA for processing or destruction by the eukaryotic RNA exosome and its cofactors. Genes Dev. 31, 88-100 (2017).

3. Mitchell, P., Petfalski, E., Shevchenko, A., Mann, M. \& Tollervey, D. The exosome: a conserved eukaryotic RNA processing complex containing multiple 3'->5' exoribonucleases. Cell 91, 457-466 (1997).

4. Makino, D. L., Halbach, F. \& Conti, E. The RNA exosome and proteasome: common principles of degradation control. Nat. Rev. Mol. Cell Biol. 14, 654-660 (2013).

5. Dziembowski, A., Lorentzen, E., Conti, E. \& Seraphin, B. A single subunit, Dis3, is essentially responsible for yeast exosome core activity. Nat. Struct. Mol. Biol. 14, 15-22 (2007).

6. Liu, Q., Greimann, J. C. \& Lima, C. D. Reconstitution, activities, and structure of the eukaryotic RNA exosome. Cell 127, 1223-1237 (2006).

7. Butler, J. S. \& Mitchell, P. Rrp6, Rrp47 and cofactors of the nuclear exosome. Adv. Exp. Med Biol. 702, 91-104 (2010).

8. Milligan, L. et al. A yeast exosome cofactor, Mpp6, functions in RNA surveillance and in the degradation of noncoding RNA transcripts. Mol. Cell Biol. 28, 5446-5457 (2008).

9. Schilders, G., Raijmakers, R., Raats, J. M. \& Pruijn, G. J. MPP6 is an exosomeassociated RNA-binding protein involved in 5.8S rRNA maturation. Nucleic Acids Res 33, 6795-6804 (2005).

10. Falk, S., Bonneau, F., Ebert, J., Kogel, A. \& Conti, E. Mpp6 incorporation in the nuclear exosome contributes to RNA channeling through the Mtr4 helicase. Cell Rep. 20, 2279-2286 (2017).

11. Schuch, B. et al. The exosome-binding factors Rrp6 and Rrp47 form a composite surface for recruiting the Mtr4 helicase. EMBO J. 33, 2829-2846 (2014).

12. Weick, E. M. et al. Helicase-dependent RNA decay illuminated by a Cryo-EM structure of a human nuclear RNA exosome-MTR4 complex. Cell 173, 1663-1677 e21 (2018).

13. Gerlach, P. et al. Distinct and evolutionary conserved structural features of the human nuclear exosome complex. Elife 7, e38686 (2018).

14. Jackson, R. N. et al. The crystal structure of Mtr4 reveals a novel arch domain required for rRNA processing. EMBO J. 29, 2205-2216 (2010).

15. Kilchert, C., Wittmann, S. \& Vasiljeva, L. The regulation and functions of the nuclear RNA exosome complex. Nat. Rev. Mol. Cell Biol. 17, 227-239 (2016).

16. Johnson, S. J. \& Jackson, R. N. Ski2-like RNA helicase structures: common themes and complex assemblies. RNA Biol. 10, 33-43 (2013).

17. Lubas, M. et al. Interaction profiling identifies the human nuclear exosome targeting complex. Mol. Cell 43, 624-637 (2011).

18. Ogami, K., Chen, Y. \& Manley, J. L. RNA surveillance by the nuclear RNA exosome: mechanisms and significance. Noncoding RNA 4, 8 (2018).

19. Vanacova, S. et al. A new yeast poly(A) polymerase complex involved in RNA quality control. PLoS Biol. 3, e189 (2005).

20. LaCava, J. et al. RNA degradation by the exosome is promoted by a nuclear polyadenylation complex. Cell 121, 713-724 (2005).

21. Wyers, F. et al. Cryptic pol II transcripts are degraded by a nuclear quality control pathway involving a new poly(A) polymerase. Cell 121, 725-737 (2005).

22. Kadaba, S., Wang, X. \& Anderson, J. T. Nuclear RNA surveillance in Saccharomyces cerevisiae: Trf4p-dependent polyadenylation of nascent hypomethylated tRNA and an aberrant form of 5S rRNA. RNA 12, 508-521 (2006).

23. Carneiro, T. et al. Depletion of the yeast nuclear exosome subunit Rrp6 results in accumulation of polyadenylated RNAs in a discrete domain within the nucleolus. Mol. Cell Biol. 27, 4157-4165 (2007).

24. Hiraishi, N., Ishida, Y. \& Nagahama, M. AAA-ATPase NVL2 acts on MTR4exosome complex to dissociate the nucleolar protein WDR74. Biochem Biophys. Res Commun. 467, 534-540 (2015).

25. Hiraishi, N., Ishida, Y. I., Sudo, H. \& Nagahama, M. WDR74 participates in an early cleavage of the pre-rRNA processing pathway in cooperation with the nucleolar AAA-ATPase NVL2. Biochem Biophys. Res Commun. 495, 116-123 (2018).

26. Gustafson, M. P., Welcker, M., Hwang, H. C. \& Clurman, B. E. Zcchc8 is a glycogen synthase kinase- 3 substrate that interacts with RNA-binding proteins. Biochem Biophys. Res Commun. 338, 1359-1367 (2005).

27. Lubas, $M$. et al. The human nuclear exosome targeting complex is loaded onto newly synthesized RNA to direct early ribonucleolysis. Cell Rep. 10, 178-192 (2015).

28. Ogami, K. et al. An Mtr4/ZFC3H1 complex facilitates turnover of unstable nuclear RNAs to prevent their cytoplasmic transport and global translational repression. Genes Dev. 31, 1257-1271 (2017).

29. Silla, T., Karadoulama, E., Makosa, D., Lubas, M. \& Jensen, T. H. The RNA exosome adaptor ZFC3H1 functionally competes with nuclear export activity to retain target transcripts. Cell Rep. 23, 2199-2210 (2018).

30. Meola, N. et al. Identification of a nuclear exosome decay pathway for processed transcripts. Mol. Cell 64, 520-533 (2016).

31. Wang, J. et al. NRDE2 negatively regulates exosome functions by inhibiting MTR4 recruitment and exosome interaction. Genes Dev 33, 536-549 (2019).

32. Weir, J. R., Bonneau, F., Hentschel, J. \& Conti, E. Structural analysis reveals the characteristic features of Mtr4, a DExH helicase involved in nuclear RNA processing and surveillance. Proc. Natl Acad. Sci. USA 107, 12139-12144 (2010).

33. Schuller, J. M., Falk, S., Fromm, L., Hurt, E. \& Conti, E. Structure of the nuclear exosome captured on a maturing preribosome. Science 360, 219-222 (2018).

34. Falk, S. et al. The molecular architecture of the TRAMP complex reveals the organization and interplay of its two catalytic activities. Mol. Cell 55, 856-867 (2014).

35. Thoms, M. et al. The exosome is recruited to RNA substrates through specific adaptor proteins. Cell 162, 1029-1038 (2015).

36. Falk, S. et al. Structural insights into the interaction of the nuclear exosome helicase Mtr4 with the preribosomal protein Nop53. RNA 23, 1780-1787 (2017).

37. Puno, M. R. \& Lima, C. D. Structural basis for MTR4-ZCCHC8 interactions that stimulate the MTR4 helicase in the nuclear exosome-targeting complex. Proc. Natl Acad. Sci. USA 115, E5506-E5515 (2018).

38. Nagahama, M. et al. The AAA-ATPase NVL2 is a component of preribosomal particles that interacts with the DExD/H-box RNA helicase DOB1. Biochem. Biophys. Res Commun. 346, 1075-1082 (2006).

39. Fujiwara, Y. et al. Structure and function of the N-terminal nucleolin binding domain of nuclear valosin-containing protein-like 2 (NVL2) harboring a nucleolar localization signal. J. Biol. Chem. 286, 21732-21741 (2011).

40. Meyer, H., Bug, M. \& Bremer, S. Emerging functions of the VCP/p97 AAAATPase in the ubiquitin system. Nat. Cell Biol. 14, 117-123 (2012).

41. Hanzelmann, P. \& Schindelin, H. The structural and functional basis of the p97/valosin-containing protein (VCP)-interacting motif (VIM): mutually exclusive binding of cofactors to the N-terminal domain of p97. J. Biol. Chem. 286, 38679-38690 (2011)

42. Buchberger, A., Schindelin, H. \& Hanzelmann, P. Control of p97 function by cofactor binding. FEBS Lett. 589, 2578-2589 (2015).

43. Falk, S. et al. Structure of the RBM7-ZCCHC8 core of the NEXT complex reveals connections to splicing factors. Nat. Commun. 7, 13573 (2016)

44. Cote, J. \& Richard, S. Tudor domains bind symmetrical dimethylated arginines. J. Biol. Chem. 280, 28476-28483 (2005).

45. Friberg, A., Oddone, A., Klymenko, T., Muller, J. \& Sattler, M. Structure of an atypical tudor domain in the drosophila polycomblike protein. Protein Sci. 19, 1906-1916 (2010).

46. Sattler, M., Schleucher, J. \& Griesinger, C. Heteronuclear multidimensional NMR experiments for the structure determination of proteins in solution employing pulsed field gradients. Prog. NMR Spectrosc. 34, 93-158 (1999).

47. Farrow, N. A. et al. Backbone dynamics of a free and phosphopeptidecomplexed Src homology 2 domain studied by 15N NMR relaxation. Biochemistry 33, 5984-6003 (1994).

48. Grzesiek, S. \& Bax, A. The importance of not saturating $\mathrm{H}_{2} \mathrm{O}$ in protein NMR - application to sensitivity enhancement and NOE measurements. J. Am. Chem. Soc. 115, 12593-12594 (1993).

49. Piotto, M., Saudek, V. \& Sklenář, V. Gradient-tailored excitation for singlequantum NMR spectroscopy of aqueous solutions. J. Biomol. NMR 2, 661 (1992). 
50. Vranken, W. F. et al. The CCPN data model for NMR spectroscopy: development of a software pipeline. Proteins 59, 687-696 (2005).

51. Winter, G. xia2: an expert system for macromolecular crystallography data reduction. J. Appl. Crystallogr. 43, 186-190 (2010).

52. Winter, G. et al. DIALS: implementation and evaluation of a new integration package. Acta Crystallogr D. Struct. Biol. 74, 85-97 (2018).

53. Potterton, L. et al. CCP4i2: the new graphical user interface to the CCP4 program suite. Acta Crystallogr. D. Struct. Biol. 74, 68-84 (2018).

54. McCoy, A. J. et al. Phaser crystallographic software. J. Appl. Crystallogr 40, 658-674 (2007).

55. Emsley, P., Lohkamp, B., Scott, W. G. \& Cowtan, K. Features and development of Coot. Acta Crystallogr. D. Biol. Crystallogr 66, 486-501 (2010).

56. Adams, P. D. et al. PHENIX: a comprehensive Python-based system for macromolecular structure solution. Acta. Crystallogr. D Biol. Crystallogr. 66, (213-221 (2010).

57. Painter, J. \& Merritt, E. A. Optimal description of a protein structure in terms of multiple groups undergoing TLS motion. Acta Crystallogr. D. Biol. Crystallogr 62, 439-450 (2006).

58. Poser, I. et al. BAC TransgeneOmics: a high-throughput method for exploration of protein function in mammals. Nat. Methods 5, 409-415 (2008).

59. Di Tommaso, P. et al. T-Coffee: a web server for the multiple sequence alignment of protein and RNA sequences using structural information and homology extension. Nucleic Acids Res 39, W13-W17 (2011).

\section{Acknowledgements}

We would like to thank the Crystallization Facility of MPI Biochemistry and also Vincent D. Maciej for his contributions at the early stages of this project. This study was supported by the Max Planck Gesellschaft, the European Commission (ERC Advanced Investigator Grant 294371) and the Deutsche Forschungsgemeinschaft (SFB646, SFB1035, GRK1721, FOR1680 to E.C., and SFB1035 and GRK1721 to M.S. This work was supported by the Cluster of Excellence EXC114 (to E.C. and M.S.). T.H.J. was supported by the ERC (grant 339953), the Lundbeck- and the Novo Nordisk-Foundations.

\section{Author contributions}

S.F and E.C. initiated the project; M.L. performed the in vitro experiments; D.J. performed the co-IP experiments under the supervision of T.H.J.; M.L. and J.B. performed the crystallography experiments, M.L. and S.F. built, refined and analyzed the structure; A.S. and M.S. collected and analyzed NMR data; L.L. performed preliminary in vitro experiments with NVL under the supervision of S.F.; M.L., S.F., and E.C. prepared the paper.

\section{Additional information}

Supplementary Information accompanies this paper at https://doi.org/10.1038/s41467019-11339-x.

Competing interests: The authors declare no competing interests.

Reprints and permission information is available online at http://npg.nature.com/ reprintsandpermissions/

Peer review information: Nature Communications thanks David Tollervey, and the other, anonymous, reviewer(s) for their contribution to the peer review of this work. Peer reviewer reports are available.

Publisher's note: Springer Nature remains neutral with regard to jurisdictional claims in published maps and institutional affiliations.

(c) (i) Open Access This article is licensed under a Creative Commons Attribution 4.0 International License, which permits use, sharing, adaptation, distribution and reproduction in any medium or format, as long as you give appropriate credit to the original author(s) and the source, provide a link to the Creative Commons license, and indicate if changes were made. The images or other third party material in this article are included in the article's Creative Commons license, unless indicated otherwise in a credit line to the material. If material is not included in the article's Creative Commons license and your intended use is not permitted by statutory regulation or exceeds the permitted use, you will need to obtain permission directly from the copyright holder. To view a copy of this license, visit http://creativecommons.org/ licenses/by/4.0/.

(c) The Author(s) 2019 